\title{
A practical method for the detection of freezing of gait in patients with Parkinson's disease
}

This article was published in the following Dove Press journal:

Clinical Interventions in Aging

8 October 2014

Number of times this article has been viewed

\author{
Yuri Kwon' \\ Sang Hoon Park' \\ Ji-Won Kim' \\ Yeji Ho' \\ Hyeong-Min Jeon' \\ Min-Jung Bang' \\ Gu-In Jung' \\ Seon-Min Lee ${ }^{3}$ \\ Gwang-Moon Eom ${ }^{1,2}$ \\ Seong-Beom Koh ${ }^{3}$ \\ Jeong-Whan Lee ${ }^{1,2}$ \\ Heung Seok Jeon ${ }^{4}$ \\ 'Biomedical Engineering, ${ }^{2}$ Research \\ Institute of Biomedical Engineering, \\ Konkuk University, Chungju, Korea; \\ ${ }^{3}$ Department of Neurology, Korea \\ University College of Medicine, Seoul, \\ Korea; ${ }^{4}$ Department of Computer \\ Engineering, Konkuk University, \\ Chungju, Korea
}

Correspondence: Gwang-Moon Eom Biomedical Engineering, Konkuk University, 322 Danwal-dong, Chungju-city, 380-70I, Korea

Tel +82438529890

Fax +824385I 0620

Email gmeom@kku.ac.kr

Seong-Beom Koh

Department of Neurology, Korea

University College of Medicine,

I48 Gurodong-ro, Guro-gu, Seoul,

152-703, Korea

Tel +82226263169

Email parkinson@korea.ac.kr
Purpose: Freezing of gait (FOG), increasing the fall risk and limiting the quality of life, is common at the advanced stage of Parkinson's disease, typically in old ages. A simple and unobtrusive FOG detection system with a small calculation load would make a fast presentation of on-demand cueing possible. The purpose of this study was to find a practical FOG detection system.

Patients and methods: A sole-mounted sensor system was developed for an unobtrusive measurement of acceleration during gait. Twenty patients with Parkinson's disease participated in this study. A simple and fast time-domain method for the FOG detection was suggested and compared with the conventional frequency-domain method. The parameters used in the FOG detection were optimized for each patient.

Results: The calculation load was 1,154 times less in the time-domain method than the conventional method, and the FOG detection performance was comparable between the two domains $(P=0.79)$ and depended on the window length $(P<0.01)$ and dimension of sensor information $(P=0.03)$.

Conclusion: A minimally constraining sole-mounted sensor system was developed, and the suggested time-domain method showed comparable FOG detection performance to that of the conventional frequency-domain method. Three-dimensional sensor information and 3-4-second window length were desirable. The suggested system is expected to have more practical clinical applications.

Keywords: Parkinson's disease, freezing of gait, acceleration, detection system, time-domain, frequency-domain

\section{Introduction}

Freezing of gait (FOG) is a common phenomenon at the advanced stage of Parkinson's disease (PD), especially in late-onset elderly patients. ${ }^{1}$ FOG is characterized by a sudden and transient inability to move during locomotion, typically at the start, turn, in tight quarters, and at the destination. ${ }^{2}$ Patients report that their feet are glued to the ground during the FOG episodes. ${ }^{2}$ Approximately $50 \%$ of PD patients suffer from FOG, specifically, $10 \%$ of patients with mild symptoms and $80 \%$ of severely affected patients regularly experience FOG. ${ }^{3,4}$ FOG is a common cause of falls,, 5 limiting daily activities and impairing quality of life. ${ }^{?}$

Pathophysiology of FOG is poorly understood. ${ }^{8,9}$ Medication is often ineffective at relieving FOG,${ }^{10}$ and the patients suffer from gradual wearing-off and also from sharp and unexpected off/on phenomenon of the medication effect. Surgical treatment such as deep brain stimulation was proposed, ${ }^{11}$ but the overall results were disappointing, ${ }^{12}$ and surgery can induce gait problems in some patients. ${ }^{13}$ Therefore, any effective non-medication and non-surgical treatment would be very helpful as subsidiary or alternative to medication. 
Presentation of external cues (cueing) such as visual, auditory, and somatosensory cueing has been shown to be effective at relieving FOG. ${ }^{14-16}$ Incidental cueing, only at the occurrence of FOG (on-demand cueing), was shown to be more effective than continuous cueing in reducing the duration of FOG episodes, ${ }^{17}$ indicating that "on-demand cueing" better directs the patient's attention back to the walking task in contrast to the intrusiveness and habituation effects of "continuous cueing" reducing efficiency. ${ }^{17}$ For the ondemand cueing, a fast detection of FOG is needed for the presentation of cueing in an appropriate time and, accordingly, for the timely unfreezing of gait. The detection system also needs to be small, light-weighted, wearable, and unobtrusive to not interfere with normal gait of the patients. ${ }^{18}$

Inspection of the trembling of the legs during $\mathrm{FOG}^{2}$ led researchers to investigate frequency characteristics of the trembling in comparison to normal walking and design frequency-domain methods for the detection of FOG. Moore et $\mathrm{l}^{19}$ defined the freeze index (FI) as the power ratio of freeze band $(0.5-3.0 \mathrm{~Hz})$ to locomotor band $(3-8 \mathrm{~Hz})$ derived from the frequency spectrum and identified FOG episodes at the time periods when FI exceeds a certain threshold. Afterwards, many researchers followed Moore's method. ${ }^{10,20}$ For example, Morris et $\mathrm{al}^{20}$ compared the identified (objective) FOG periods (with the help of FI) with the clinical gold standard and found strong agreement between them. Bachlin et $\mathrm{al}^{10}$ utilized the total power, in addition to Moore's method, to differentiate FOG episodes from the standing period.

The major limitation of the frequency-domain methods is that the amount of calculations needed for Fourier transform is large, and a digital signal processing chip is required, hence the algorithm is hard to be implemented in a small, light-weighted, and inexpensive system. For the patients with gait problems, a bulky and obtrusive system may worsen the gait disturbances. Besides, relatively long window length (4 seconds, ${ }^{10} 6$ seconds, ${ }^{19}$ and 10 seconds ${ }^{20}$ ) was used for the detection, leading to long delay in the FOG detection.

Therefore, if a simpler method is available with a performance comparable to that of the frequency-domain method, it would be advantageous for the wearable hardware implementation. From the observation that the accelerations of the foot reflecting the "trembling during FOG" has a typical amplitude range, we hypothesized that the amplitude of the leg acceleration in the time domain can differentiate FOG episodes from the others such as standing and walking. Therefore, one purpose of this study was to suggest a time-domain method for the detection of FOG and compare its performance with that of the frequency-domain method.
Another objective was to develop a minimally constraining system for the measurement of leg acceleration during gait. The system was developed by mounting a sensor in the sole and wireless transmission of data.

\section{Patients and methods Measurement system}

Attachment of sensors on the body part (shank, thigh, and lower back) has been adopted in the literature; ${ }^{10,18-21}$ however, it may burden patients and constrain locomotion, worsening gait abnormalities. Therefore, a non-constraining measurement system is desirable. We observed that the "trembling" of the legs during FOG is reflected on the acceleration of foot and shoe, especially on the heel (Figure 1). During FOG, acceleration signals become smaller than those during normal gait. This has also been shown by the fluctuation of sole forces observed during FOG, where the forces reflect the leg and body accelerations. ${ }^{8}$

Therefore, we decided to develop a minimally constraining measurement system with the accelerometer built in a shoe. Specifically, a miniature printed circuit board (PCB) including three-axes accelerometer (MMA7260Q; Freescale Inc., Austin, TX, USA) and a microprocessor was developed, and it was inserted in the heel part of a shoe-sole, beneath the insole (Figure 2A). A shallow rectangular hole was carved in the sole just beneath the heel, where the PCB of the accelerometer unit was firmly fixed. Then, the PCB as well as the sole was covered by an insole. For the good coordination of foot and shoe, the foot was fixed in the shoe by the Velcro-fastener on the top of the shoe. Therefore, the acceleration of the shoe coming from the foot was directly measured by the sensor.

Measured signals may be processed online by the microprocessor if the FOG detection algorithm and parameters are pre-determined. In this study, however, the sensor signal was saved in a personal computer (PC) and processed offline to test various FOG detection methods. Sampling frequency was $250 \mathrm{~Hz}$, and the sampled data was transmitted to a PC via wireless (Bluetooth) communication (Figure 2B).

\section{Subjects and experiments}

Twenty patients with PD who reported the FOG history during daily activities were included in this study. The study was approved by the Institutional Review Board and was performed in accordance with the ethical standards of the Declaration of Helsinki. All subjects provided informed written consent, and all of them were in the on-medication state during the experiments for ethical reasons - overnight 


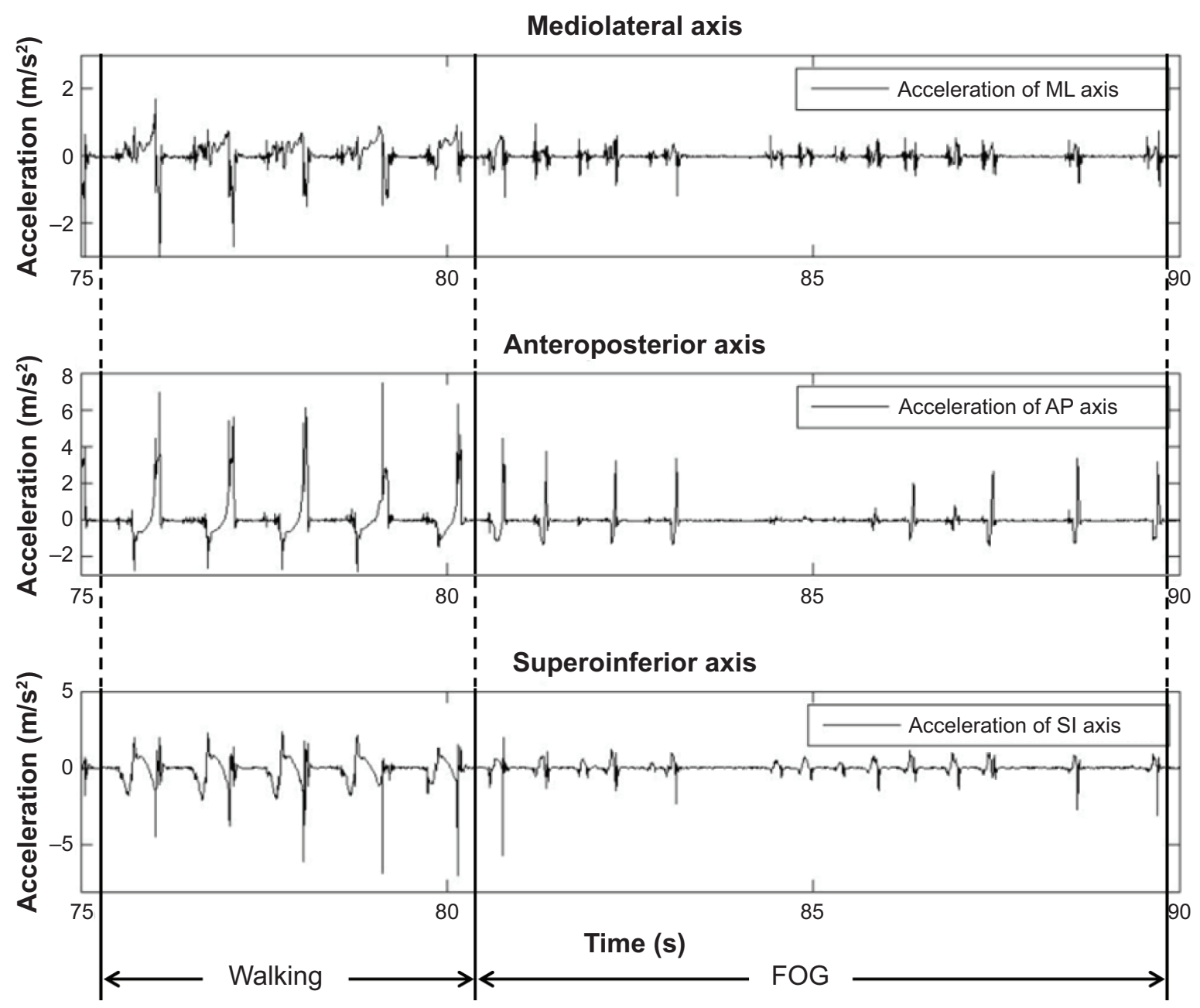

Figure I Acceleration signals measured at the sole (heel part).

Note: The amplitude of acceleration in all three directions is smaller during FOG than during normal walking.

Abbreviations: AP, anteroposterior; FOG, freezing of gait; $\mathrm{ML}$, mediolateral; SI, superoinferior.

withdrawal of medication and coming to hospital for the experiments was considered to be dangerous for the patients and burdensome to their family.

The patients wore the instrumented shoes (the size was selected by the patients) and walked back and forth five times in a straight $6 \mathrm{~m}$ line along a hallway, including $180^{\circ}$ turns. The patients walked at their own pace without assistance, but medical staff stayed close to the patients for safety reasons. All trials were recorded on a digital video camera synchronized with the acceleration signals from the shoes, using an LED (light-emitting diode) light turned-on by the data-acquisition system. An assistant with a video camera followed close to a patient within $1 \mathrm{~m}$ distance for the legs to get a clear reflection on the video and for the FOG episodes to be easily recognized from the video.

After the measurements, a medical doctor manually identified FOG events from the careful investigation of the video recordings and registered the exact start and end timings of FOG episodes. The identified FOG periods were set as gold standards for the evaluation of automatic detection algorithms. In this study, six patients showed FOG during the test. Table 1 shows the patients' characteristics and FOG episodes.

Tests with the developed measurement system were performed for all the subjects. Because the purpose of this study was to evaluate FOG detection methods, the subsequent analysis included only the six patients' data. FOG is highly sensitive to environmental triggers, cognitive input, and medication; therefore, FOG occurs frequently at home and much less frequently in a hospital or in a gait laboratory. ${ }^{22}$ Fast $360^{\circ}$ turns are often better to elicit FOG than normalspeed $180^{\circ}$ turns. ${ }^{23}$ In the on-medication state, observing FOG in an experimental setup is more difficult. However, experiments with normal speed of $180^{\circ}$ under the on-medication state were forced by the request of the Institutional Review Board caring for the safety of patients. Six patients' data 
A

Measurement system

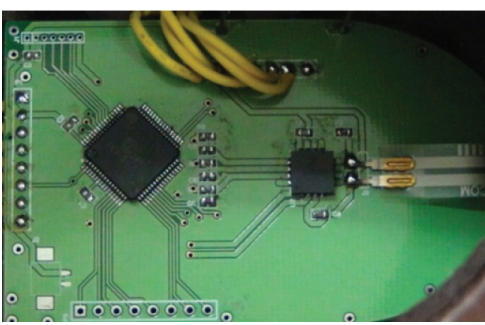

Accelerometer module

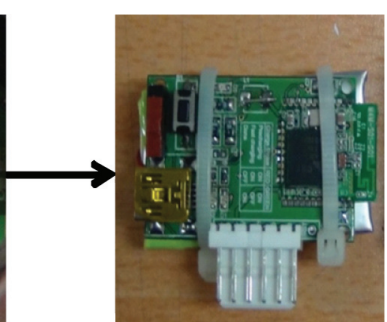

Bluetooth module

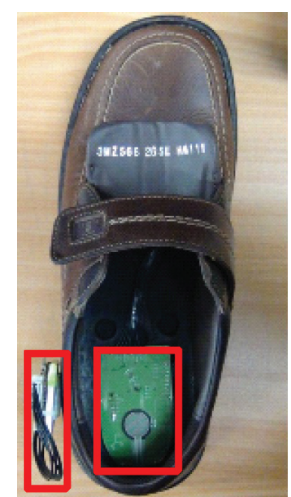

Bluetooth Acc

module module
B

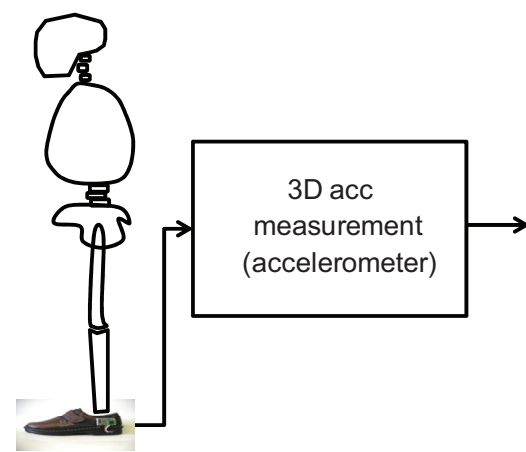

Signal flow and analysis



Figure 2 Experimental setup.

Notes: Measured signals at the accelerometer module (mounted at sole) were transmitted to a PC through Bluetooth module at the back-lateral side of the shoe (A). Transmitted data was saved by custom-made Labview software and analyzed by a custom-made Matlab program (B).

Abbreviations: 3D, three-dimensional; PC, personal computer; acc, acceleration.

Table I Subject characteristics

\begin{tabular}{|c|c|c|c|c|c|c|c|}
\hline $\begin{array}{l}\text { Subject } \\
\text { number }\end{array}$ & Sex & $\begin{array}{l}\text { Age, } \\
\text { years }\end{array}$ & $\begin{array}{l}\text { Disease } \\
\text { duration, years }\end{array}$ & $\begin{array}{l}\text { H\&Y } \\
\text { stage }\end{array}$ & $\begin{array}{l}\text { FOG } \\
\text { type }\end{array}$ & $\begin{array}{l}\text { Number of } \\
\text { FOG episodes }\end{array}$ & $\begin{array}{l}\text { Total period of } \\
\text { FOG, seconds }\end{array}$ \\
\hline 1 & $\mathrm{~F}$ & 70 & 13 & 2.5 & & & \\
\hline $2^{\mathrm{a}}$ & $M$ & 71 & 5 & 2.5 & $\mathrm{~T}$ & 9 & 28.1 \\
\hline $3^{a}$ & $M$ & 64 & 5 & 2 & $S, T$ & 4 & 107.3 \\
\hline 4 & $F$ & 78 & 10 & 2.5 & & & \\
\hline 5 & $\mathrm{~F}$ & 73 & 4 & 2.5 & & & \\
\hline 6 & $\mathrm{~F}$ & 77 & I & 3 & & & \\
\hline 7 & $M$ & 74 & I & 2.5 & & & \\
\hline 8 & $M$ & 45 & 20 & 2 & & & \\
\hline 9 & $M$ & 52 & 15 & 2.5 & & & \\
\hline 10 & $M$ & 82 & 1 & 2.5 & & & \\
\hline 11 & $\mathrm{~F}$ & 75 & 0.3 & 2.5 & & & \\
\hline 12 & $F$ & 77 & 6 & 3 & & & \\
\hline 13 & $\mathrm{~F}$ & 71 & 12 & 2.5 & & & \\
\hline 14 & $M$ & 76 & 3 & 2.5 & & & \\
\hline 15 & $M$ & 46 & 9 & 4 & & & \\
\hline $16^{a}$ & $\mathrm{~F}$ & 71 & 10 & 3 & S, T, D & 6 & 52.3 \\
\hline $17^{\mathrm{a}}$ & $M$ & 64 & 15 & 3 & $\mathrm{~S}, \mathrm{~T}, \mathrm{D}$ & 7 & 70.2 \\
\hline 18 & M & 59 & 8 & 2.5 & & & \\
\hline $19^{a}$ & $\mathrm{~F}$ & 48 & 2 & 2 & $\mathrm{~N}$ & 3 & 11.6 \\
\hline $20^{a}$ & $M$ & 64 & 2 & 3 & $\mathrm{~S}, \mathrm{~T}$ & 7 & 39.7 \\
\hline Mean \pm SD & & $66.9 \pm 11.3$ & $7.1 \pm 5.7$ & $2.6 \pm 0.5$ & & $6.0 \pm 2.2$ & $52.5 \pm 33.9$ \\
\hline
\end{tabular}

Note: ${ }^{\mathrm{F} O G}$ occurred.

Abbreviations: D, destination hesitation; F, female; FOG, freezing of gait; H\&Y, Hoehn and Yahr; M, male; N, narrow corridor passage hesitation; S, starting hesitation; $\mathrm{SD}$, standard deviation; T, turning hesitation. 
out of 20 subjects in the on-medication state of this study were assumed to be acceptable for the evaluation of FOG detection performance, considering that similar number of patients with FOG were recruited even in the off-medication state in the literature (seven patients out of ten in the Moore et al study, ${ }^{19}$ eight patients out of ten in the Bachlin et al study, ${ }^{10}$ and an unspecified number of patients out of ten in the Morris et al study ${ }^{20}$ ).

\section{Detection of FOG and statistical analysis}

Detection of FOG can be performed online, once the algorithm and parameters are determined. The online detection would be the "post-freezing" identification as soon as FOG occurs. Because the purpose of this study was to evaluate various FOG detection methods, the performance of each method was analyzed offline with an identical dataset.

We applied two categories of automated algorithms for the detection of FOG. One was the time-domain method, and the other was the frequency-domain method introduced by Bachlin et al. ${ }^{10}$ The performance of the FOG detection was defined as the minimum of sensitivity (actual FOG detected as FOG) and specificity (actual non-FOG detected as non-FOG), because both sensitivity and specificity were assumed to be important. Sensitivity and specificity were calculated as the ratio of time durations rather than the ratio of number of events.

When multiple parameters are required, the optimization of the parameters to get the best performance is common.
For example, Bachlin et a ${ }^{10}$ optimized two parameters through the inspection of three-dimensional (3D) representation of the performance with respect to two-dimensional parameters. In the current study, the parameters in each method were optimized for each subject to maximize the performance, because locomotion and freezing patterns differ among patients. The optimization was performed by the simulated annealing method implemented in Matlab (Mathworks, Natick, MA, USA). The maximum number of cost function evaluations for an optimization in one condition was set as 20,000 by trial and error.

In the suggested time-domain methods, one-dimensional (1D) (vertical) acceleration signal or all 3D signals were used for the FOG detection to investigate the dependence of performance on the dimension of information. From the observation that the "trembling" of the legs during the FOG is reflected on acceleration signals with typical amplitude range, we hypothesized that the root-mean-squared (RMS) mean of the acceleration can differentiate FOG episodes from the others (ie, medium-range RMS acceleration would represent trembling of the leg in the FOG, differentiated from a too low value representing static (standing) period and a too high value representing a dynamic gait (walking) period as shown in Figure 3). The RMS mean of each axis acceleration was calculated for a window (with the number of data, $N$, depending on the window length) sliding with 0.1 second time step throughout the whole data. If the RMS mean of a window was determined to be in the FOG range, the central

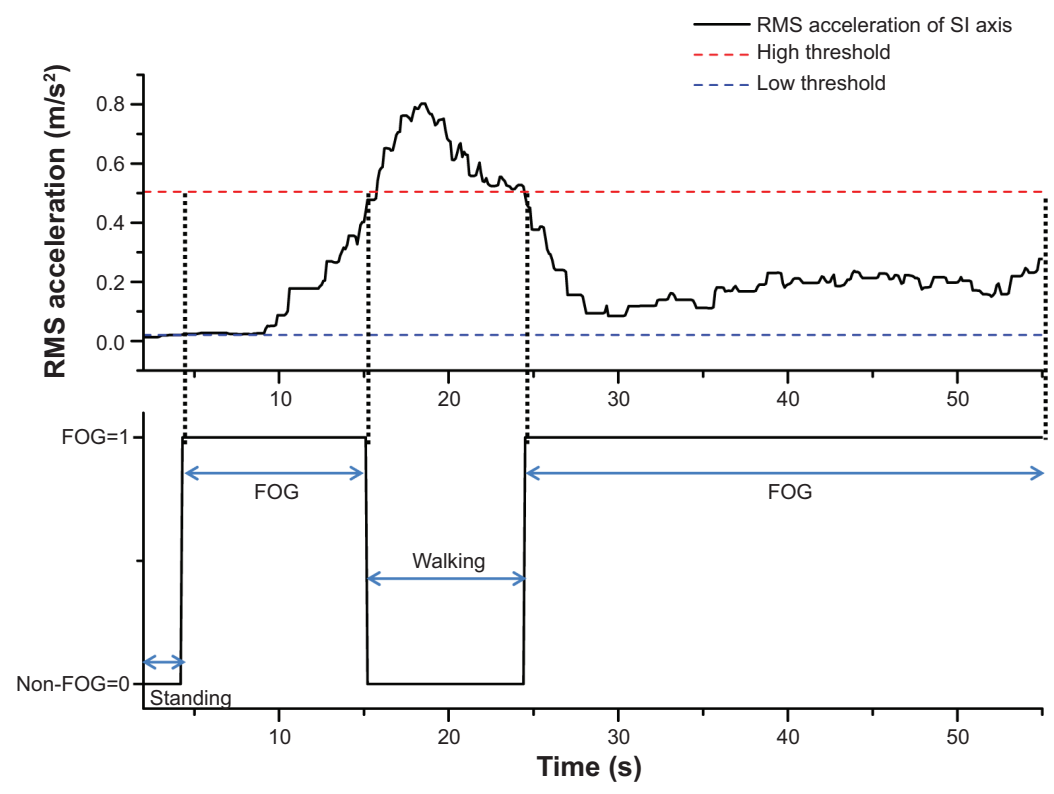

Figure 3 FOG detection in time domain (ID signal).

Notes: FOG periods are characterized by RMS acceleration of the medium range between two thresholds. RMS acceleration below the low threshold represents the standing period. Likewise, RMS acceleration above the high threshold represents the walking period.

Abbreviations: ID, one-dimensional; FOG, freezing of gait; RMS, root-mean-squared; SI, superoinferior. 
time instant of the window was recorded as to be in the FOG state. In the case of $1 \mathrm{D}$ signal, FOG range was defined as the range between two thresholds (low and high) as in Figure 3. In the case of 3D signals, six thresholds were required, and three FOG states (FOG: 1, non-FOG: 0) were "AND" operated to determine final FOG. This process was repeated for every sliding window to generate FOG periods.

For comparison, the frequency-domain method was also tested, where the time instant was recognized as FOG if both the FI and total power in the sliding window exceed thresholds (Figure 4). FI was defined as the ratio of power in the freeze band $(0.5-3.0 \mathrm{~Hz})$ to locomotion band $(3-8 \mathrm{~Hz})$ resulting in the increased FI value during FOG episodes. ${ }^{10,19}$ Total power was included to rule out the resting (standing) period from FOG (ie, a low total power indicates that the patient is not moving at all; therefore, it cannot be regarded as FOG irrespective of FI). The parameters to be optimized were two thresholds (for FI and total power) for 1D signal and six thresholds for 3D signals. As in the case of the timedomain method, three FOG states were "AND" operated to determine final FOG when 3D signals were included.

The performance of FOG detection would depend on the window length; therefore, the performance was investigated for eight different window lengths in the range $0.2-8.0$ seconds $(0.2,1.0,2.0,3.0,3.5,4.0,4.5,6.0$, and 8 seconds). The window was moved throughout the whole data of each subject with 0.1 second time step, resulting in the 0.1 second temporal resolution of the detected FOG.

Three-way analysis of variance (ANOVA) was performed on the performance (the minimum of sensitivity and specificity) with factors of the window length (0.2-8.0 seconds), analysis domain (time vs frequency), and dimension of sensor data (1D vs 3D). The level of significance was defined as $P<0.01$. All statistical analyses were performed using SPSS version 16 (SPSS Inc., Chicago, IL, USA).

\section{Amount of calculations in the automatic FOG detections}

In this section, the calculation load in the two domain methods were estimated and compared, because smaller load is important for the practicality of a FOG detection system, as explained in the introduction.

Multiplication is the main component of time-consumption on general purpose computers; ${ }^{24}$ therefore, the amount of calculations can be represented roughly by the number of multiplications. The calculation time of a square-root and

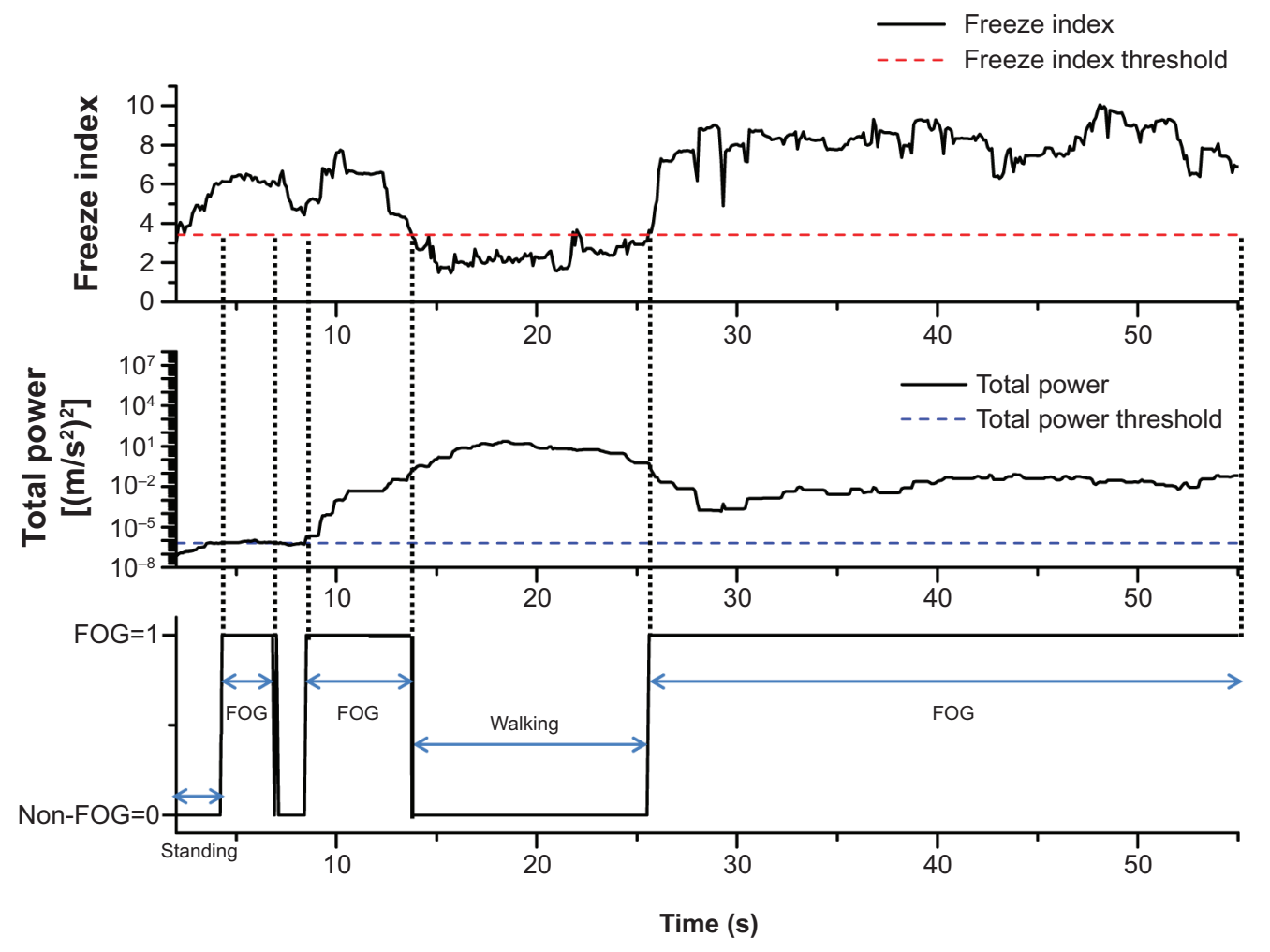

Figure 4 FOG detection in frequency domain (ID signal).

Notes: If both the freeze index and total power are greater than each threshold, the periods were classified as FOG episodes. Standing period was characterized by small total power (below the threshold). Walking period was characterized by low freeze index (below threshold) with enough total power (above the threshold).

Abbreviations: ID, one-dimensional; FOG, freezing of gait. 
division was replaced by the comparable number of realnumber multiplications (two and 12 times, respectively), which was investigated from an experiment on a PC (CPU: Intel ${ }^{\circledR}$ Core $^{\mathrm{TM}}$ i7-2600 CPU @ 3.4 GHz. Memory: 4 GB). Operations with smaller calculation load (addition, subtraction, and other control operations) were disregarded for the estimate to be only approximate yet valuable. ${ }^{25}$

The number of data in a sliding window was designated as $N$, and the step length of sliding window was designated as $N_{1}(0.1$ second $\times 250 \mathrm{~Hz}=25$ samples in this study $)$. In the case of $3 \mathrm{D}$ signals, the ratio of calculation load in the two domain methods would just be identical to that of $1 \mathrm{D}$; therefore, only $1 \mathrm{D}$ case is described, as follows.

The frequency-domain method requires a frequency spectrum to calculate the FI and total power. To get the frequency spectrum, Fourier transform is needed and fast version of Fourier transform results in $N \log _{2} N$ complex multiplications. ${ }^{24}$ Total power and FI need square calculation of the amplitude for all digital frequencies, resulting in $N$ complex multiplications. Overall, $N\left(1+\log _{2} N\right)$ complex multiplications are needed. One complex multiplication can be represented by four times of real multiplications. Finally, the frequency-domain method requires $4 N\left(1+\log _{2} N\right)$ real multiplications.

The time-domain method requires RMS calculation, which requires $N$ multiplications, one division and one square-rooting so that the calculation load of RMS is equivalent to that of $N+14$ multiplications. RMS calculation at the second sliding window is displaced $N_{1}$ samples from the initial window. The squares of the samples for $n=N_{1}+1$ to $N$ were already calculated in the previous (first) window; therefore, their sum can be saved into memory as $M$ for the use in the second window. Therefore, in the second window, the number of square operations is reduced to newly added $N_{1}$ samples. Now, the total number of multiplication is $N_{1}+14$ including division and square-rooting, and this can be applied to all the subsequent windows.

Then, the frequency-to-time load ratios, which is equivalent to the speed advantage of the time-domain method, was derived. The window length of 4 seconds was used for this calculation, because the best detection performance was achieved with 4 seconds of sliding window in most cases (Figure 5 and Table 2). When substituting $N$ by 1,000 (4 seconds $\times 250 \mathrm{~Hz}$ ) and $N_{1}$ by $25(0.1$ second $\times 250 \mathrm{~Hz}$ ), the load ratio was 43 at the first window and 1,154 at the second and subsequent windows. In the actual usage, the first window is used only once, and all the subsequent iterations are ruled by the load ratio 1,154 . This indicates that the amount of calculations is dramatically reduced by adopting the time-domain method. Therefore, if the performance of the time-domain method is comparable to that of the frequencydomain method, the time-domain method would have far better practicality.

\section{Results}

Figure 6 shows the representative result of the FOG detection with 4-second sliding window length. The frequency-domain method shows many fluctuations of FOG states in contrast to stable FOG states in the time-domain methods.

Table 2 shows the FOG detection performance for all three factors (ie, the analysis domain, dimension of sensor information, and length of sliding window). Figure 5 shows the performance of four methods (two domains $x$ two information dimensions) averaged for six patients who showed FOG during the experiments. Figure 5 also shows the performance of each method in each of six patients. The performance was shown with respect to window length.

Because the cost function was set as the performance (minimum of sensitivity and specificity), both the sensitivity and specificity were similarly improved by the optimization (simulated annealing), as shown in Figure 7.

Table 3 shows the result of three-way ANOVA for each of the performance (minimum of sensitivity and specificity), sensitivity, and specificity. The FOG detection performance was comparable between two domains ( $P=0.794)$. The timedomain method tended to be better than the frequency-domain method (in the case of 3D information). Sensor information dimension significantly affected the performance $(P=0.030)$, where $3 \mathrm{D}$ information resulted in better performance than that of $1 \mathrm{D}$ information. The window length also significantly affected the performance $(P<0.001)$. The peak performances were shown at window length in the range 3.0-4.5 seconds. The interaction of three factors was insignificant $(P=0.730)$; therefore, no post hoc analysis was performed. As expected, all the statistical results for the sensitivity and specificity were similar to those of the performance.

\section{Discussion Effect of information dimension and window length}

As expected, the usage of all three directional accelerations resulted in significantly better performance compared with when using only one-directional (vertical) acceleration $(P=0.030)$. This study clearly indicated that more sensor information can enhance the performance of FOG detection, which is valuable for the clinical application. 

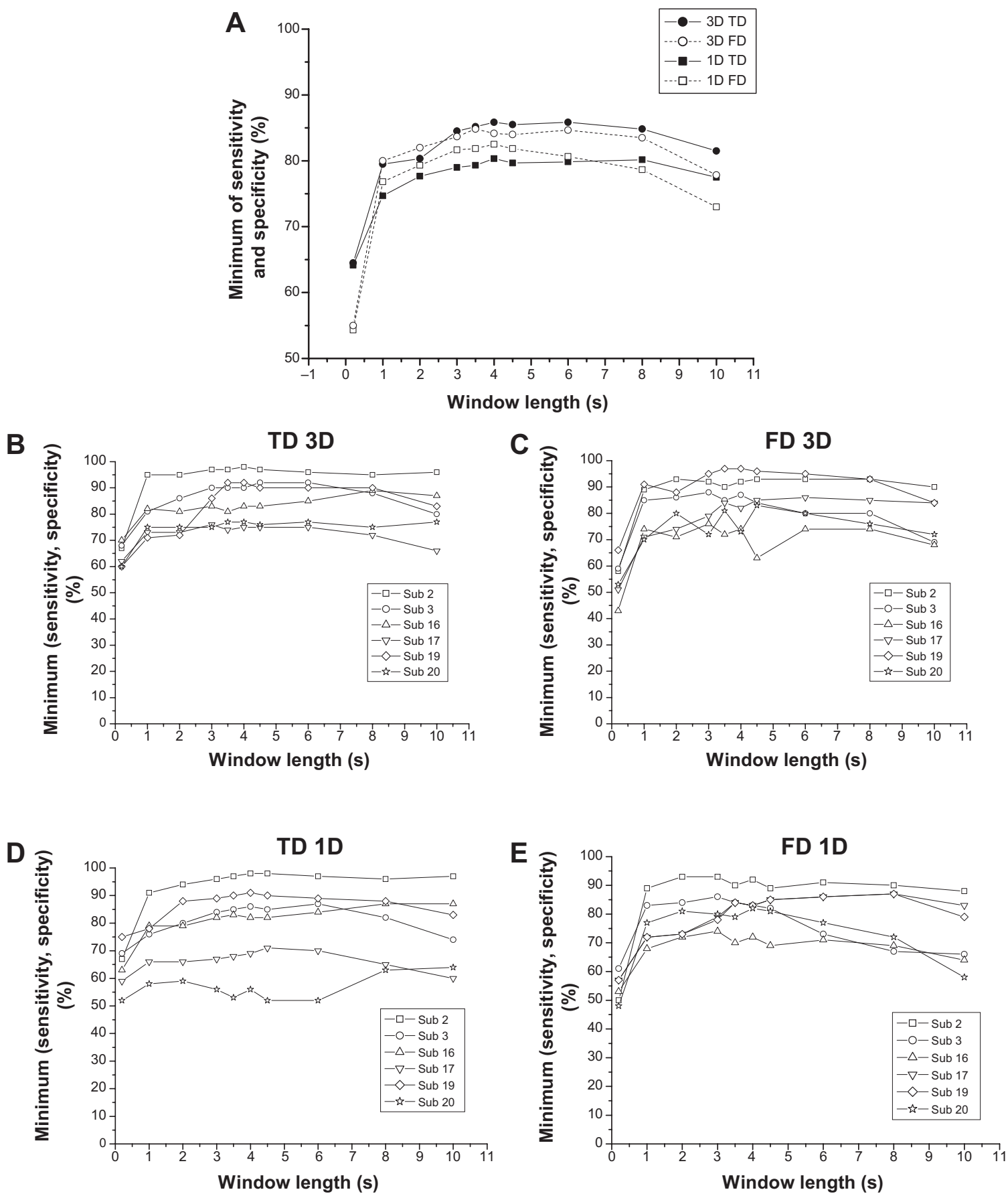

Figure 5 Average performance of each algorithm averaged for six patients who showed FOG during experiments (A), and the performance in each of six patients (B-E). Notes: The FOG detection performance was comparable between two domains. 3D sensor information significantly improved the performance. The window length also significantly affected the performance.

Abbreviations: ID, one-dimensional; 3D, three-dimensional; FD, frequency domain; FOG, freezing of gait; Sub, subject; TD, time domain.

The performance showed peaks at the window length in the range 3.0-4.5 seconds (Figure 5 and Table 2). Shorter and longer window length resulted in inferior performance. Taking the gait cycle ( $\sim 1$ second) into consideration, too short window length may result in severe fluctuation of the acceleration during a normal gait. Similarly, too long window length ( $>4$ seconds) acts as a low-pass filter and may dilute the FOG patterns (existing for a certain period of time) into a wider time window, resulting in more missed FOG events of short duration (lower sensitivity). Moreover, longer window length is associated with more delay (latency) in the online application of the algorithm in the real-world situation. Therefore, a shorter window length with acceptable performance is preferable. In this context, window length in the range of 3-4 seconds would be optimal considering both the detection accuracy and detection delay, and too long 
Table 2 FOG detection performance (minimum of sensitivity and specificity in \%)

\begin{tabular}{|c|c|c|c|c|}
\hline \multirow{2}{*}{$\begin{array}{l}\text { Window } \\
\text { length (s) }\end{array}$} & \multicolumn{2}{|c|}{ One dimensional } & \multicolumn{2}{|c|}{ Three dimensional } \\
\hline & TD & FD & TD & FD \\
\hline 0.2 & $64.2 \pm 8.1$ & $54.3 \pm 4.9$ & $64.5 \pm 4.4$ & $55.0 \pm 7.9$ \\
\hline I & $74.7 \pm I I .4$ & $76.8 \pm 7.9$ & $79.5 \pm 8.8$ & $80.0 \pm 9.4$ \\
\hline 2 & $77.7 \pm 13.2$ & $79.3 \pm 8.3$ & $80.3 \pm 8.9$ & $82.0 \pm 8.5$ \\
\hline 3 & $79.0 \pm 14.8$ & $81.7 \pm 6.8$ & $84.5 \pm 8.4$ & $83.7 \pm 9.3$ \\
\hline 3.5 & $79.3 \pm 16.1$ & $81.8 \pm 6.8$ & $85.2 \pm 9.2$ & $84.8 \pm 8.4$ \\
\hline 4 & $80.3 \pm 15.4$ & $82.5 \pm 6.3$ & $85.8 \pm 9.0$ & $84.2 \pm 9.7$ \\
\hline 4.5 & $79.7 \pm 16.2$ & $81.8 \pm 6.9$ & $85.5 \pm 9.0$ & $84.0 \pm 11.6$ \\
\hline 6 & $79.8 \pm 16.2$ & $80.7 \pm 8.1$ & $85.8 \pm 8.4$ & $84.7 \pm 8.2$ \\
\hline 8 & $80.2 \pm 13.3$ & $78.7 \pm 10.4$ & $84.8 \pm 9.2$ & $83.5 \pm 8.3$ \\
\hline 10 & $77.5 \pm 14.2$ & $73.0 \pm 12.0$ & $81.5 \pm 10.1$ & $77.8 \pm 9.3$ \\
\hline Mean \pm SD & $77.2 \pm 4.9$ & $77.1 \pm 8.5$ & $81.8 \pm 6.5$ & $80.0 \pm 9.1$ \\
\hline
\end{tabular}

Abbreviations: FD, frequency domain; FOG, freezing of gait; SD, standard deviation; TD, time domain.

window length (6-10 seconds) used in the literature ${ }^{19,20}$ needs to be avoided. When one assumes the fastness of detection to be more important than the performance, window length in the range 1-2 seconds should be used.

\section{Practicality of the time-domain method}

FOG detection performance was comparable between the time and frequency-domain methods $(P=0.794)$. When the window length was fixed, the amount of information depended only on the dimension of sensor signals, which were identical in both the methods. Moreover, the number of thresholds needed to determine FOG was identical in both the domain methods, and their functional role was similar. Specifically, the lower threshold in time-domain was used to exclude standing episodes, which was functionally equivalent to the total power threshold in the frequency-domain method. Similarly, the upper threshold in time-domain was used to exclude normal walking episodes, which were functionally equivalent to the FI threshold in the frequency-domain method. Therefore, the minor difference in the performance of the two domain methods may have resulted from the difference in signal processing and the optimality of the final thresholds. For example, the time-domain method tended to be better than the frequency-domain method when $3 \mathrm{D}$ accelerations were used, probably because of the difference in signal processing (eg, the RMS acceleration better contrasted the FOG features with locomotion features than the power ratio of FOG band to the locomotion band [FI] did). The simple calculation of the time-domain method might have resulted in the shape of the solution space being simpler than that of the frequency-domain method, and the chance of getting to global optimum in the iteration limit may have increased.

In the last section of "Material and methods," the speed advantage of the time-domain method vs the frequencydomain method was estimated as to be $>1,000$. The results showed that the performance of the time-domain method was comparable to that of the frequency-domain method. Therefore, the time-domain method would be far more practical than the frequency-domain method, because time-domain method has far less calculation load and comparable performance.

Both the time-domain and frequency-domain methods require a $>3$-second window length for accurate

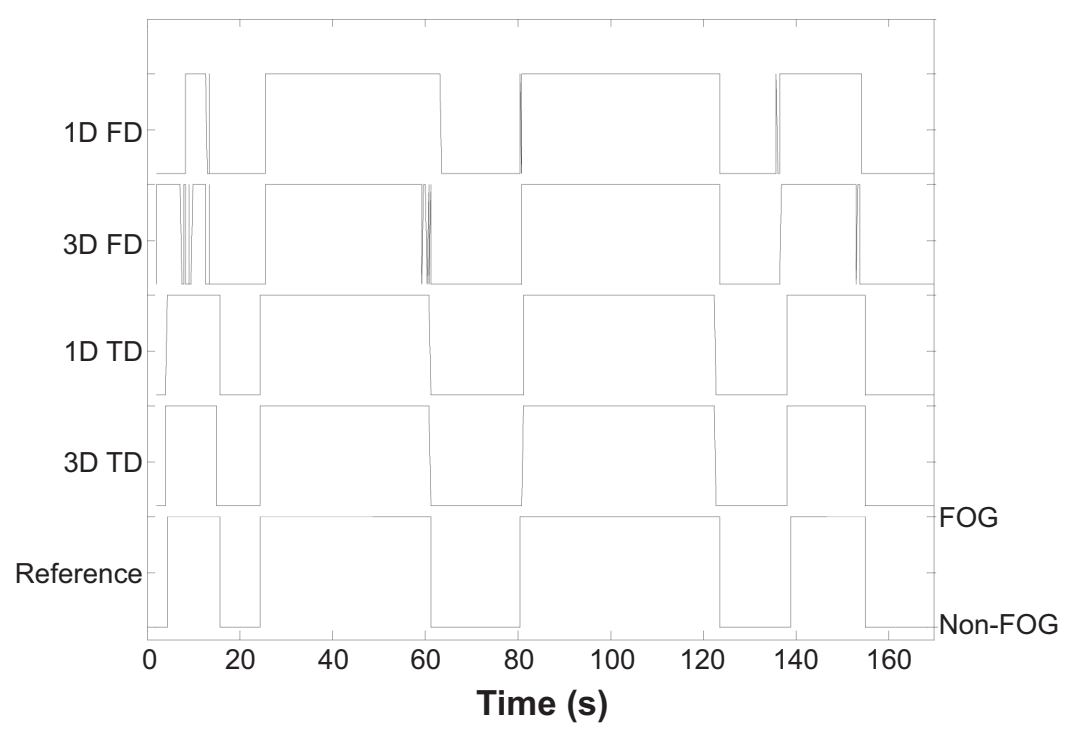

Figure 6 Representative result of FOG detection (window length $=4$ seconds).

Note: Frequency domain methods show many fluctuations of FOG states, contrasted by stable FOG states in the time-domain methods. Abbreviations: ID, one-dimensional; 3D, three-dimensional; FD, frequency domain; FOG, freezing of gait; TD, time domain. 

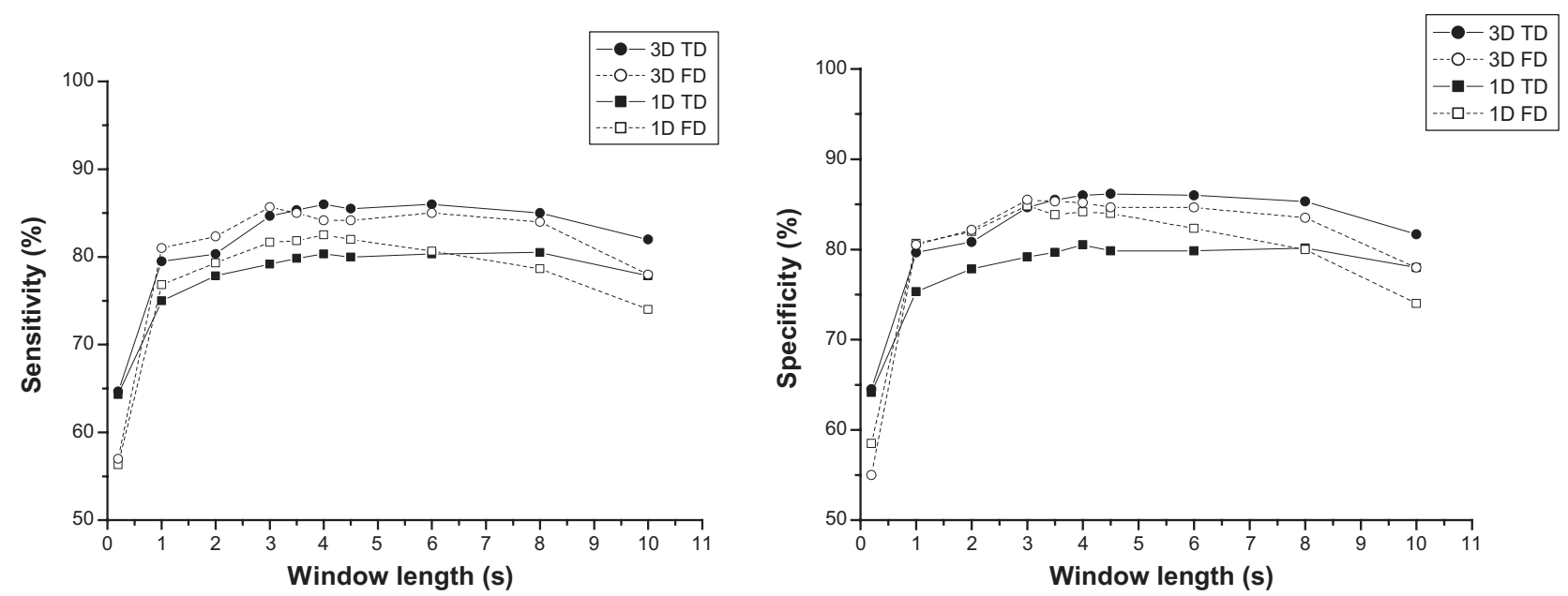

Figure 7 Sensitivity and specificity.

Note: The effect of analysis domain, sensor information dimension, and the window length on the sensitivity and specificity were similar to those on the performance (minimum of sensitivity and specificity).

Abbreviations: ID, one-dimensional; 3D, three-dimensional; FD, frequency domain; TD, time domain.

identification of freezing events, indicating that $>1.5$ seconds of delay is essential when the methods are implemented in real time (the central time of the window corresponds to the current time of the output). In this regard, faster calculations may not lead to significant improvements. Instead, an important improvement of the time-domain method would be the potential reduction in size, weight, and cost of the equipment necessary for calculations because of the reduced calculation load and ability to dispense with additional digital signal processing hardware.

\section{Further considerations}

As the cost function of optimization, the minimum of sensitivity and specificity was used under the assumption that both factors are equally important, as in the literature.$^{10}$ However, one may assume that over-detections matter less than misdetections, in which case sensitivity would be more important than the specificity. The cost function may be modified as the weighted sum of sensitivity and specificity according to the priority of each user (patient) or medical staff.

In the case of online application, only one optimization of the parameters with experimental data is needed for each subject, and the determined parameters will be used as for the FOG detection. The optimization is necessary for the

Table 3 Three-way ANOVA results ( $P$-values)

\begin{tabular}{lllll}
\hline Criteria & Domain & Dimension & Window length & Interaction \\
\hline Minimum & 0.794 & $0.030^{*}$ & $0.000^{* *}$ & 0.730 \\
Sensitivity & 0.803 & $0.006^{*}$ & $0.000^{* *}$ & 1.000 \\
Specificity & 0.629 & $0.046^{*}$ & $0.000^{* *}$ & 1.000 \\
\hline
\end{tabular}

Notes: $* P<0.05 ; * * P<0.001$.

Abbreviation: ANOVA, analysis of variance. best performance in each subject, but it may reduce the practicality of the suggested methods. To avoid this, generic parameters can be determined from multiple subjects' data, and they can be used for any patient with minor tuning through clinical trials.

\section{Conclusion}

We developed a sole-mounted measurement system and a time-domain method for the detection of FOG. The measurement system was unobtrusive and comfortable. The time-domain method showed a significantly smaller amount of calculations $(>1,000)$ than the conventional method, and its performance was comparable to the conventional method. 3D sensor information and window length in the range of 3-4 seconds are recommended. The integration of the FOG detection system with cueing modalities is further needed for the implementation of on-demand cueing.

\section{Acknowledgment}

This study was supported by the National Research Foundation (NRF) funded by the Ministry of Education, Korea (No. 2011-0015824).

\section{Disclosure}

The authors report no conflicts of interest in this work.

\section{References}

1. Contreras A, Grandas F. Risk factors for freezing of gait in Parkinson's disease. J Neurol Sci. 2012;320(1-2):66-71.

2. Schaafsma JD, Balash Y, Gurevich T, Bartels AL, Hausdorff JM, Giladi N. Characterization of freezing of gait subtypes and the response of each to levodopa in Parkinson's disease. Eur J Neurol. 2003;10(4):391-398. 
3. Giladi N. Freezing of gait. Clinical overview. Adv Neurol. 2001;87: 191-197.

4. Macht M, Kaussner Y, Moller JC, et al. Predictors of freezing in Parkinson's disease: a survey of 6,620 patients. Mov Disord. 2007;22(7): 953-956.

5. Bloem BR, Hausdorff JM, Visser JE, Giladi N. Falls and freezing of gait in Parkinson's disease: a review of two interconnected, episodic phenomena. Mov Disord. 2004;19(8):871-884.

6. Kerr GK, Worringham CJ, Cole MH, Lacherez PF, Wood JM, Silburn PA. Predictors of future falls in Parkinson disease. Neurology. 2010;75(2): 116-124.

7. de Boer AG, Wijker W, Speelman JD, de Haes JC. Quality of life in patients with Parkinson's disease: development of a questionnaire. J Neurol Neurosurg Psychiatry. 1996;61(1):70-74.

8. Hausdorff JM, Balash Y, Giladi N. Time series analysis of leg movements during freezing of gait in Parkinson's disease: akinesia, rhyme or reason? Physica A. 2003;321(3/4):565-570.

9. Danoudis M, Iansek R, Simpson P. Freezing of gait in Parkinson's disease: further insights into pathophysiological mechanisms. Parkinsonism Relat Disord. 2012;18(5):543-547.

10. Bachlin M, Plotnik M, Roggen D, et al. Wearable assistant for Parkinson's disease patients with the freezing of gait symptom. IEEE Trans Inf Technol Biomed. 2010;14(2):436-446.

11. Thevathasan W, Coyne TJ, Hyam JA, et al. Pedunculopontine nucleus stimulation improves gait freezing in Parkinson disease. Neurosurgery. 2011;69(6):1248-1253; discussion 1254.

12. Ferraye MU, Debu B, Fraix V, et al. Effects of pedunculopontine nucleus area stimulation on gait disorders in Parkinson's disease. Brain. 2010 133(Pt 1):205-214.

13. Ferraye MU, Debu B, Fraix V, et al. Effects of subthalamic nucleus stimulation and levodopa on freezing of gait in Parkinson disease. Neurology. 2008;70(16 Pt 2):1431-1437.

14. Nieuwboer A, Kwakkel G, Rochester L, et al. Cueing training in the home improves gait-related mobility in Parkinson's disease: the RESCUE trial. J Neurol Neurosurg Psychiatry. 2007;78(2):134-140.
15. Cohen RG, Horak FB, Nutt JG. Peering through the FoG: visual manipulations shed light on freezing of gait. Mov Disord. 2012;27(4): 470-472.

16. Bryant MS, Rintala DH, Lai EC, Protas EJ. A pilot study: influence of visual cue color on freezing of gait in persons with Parkinson's disease. Disabil Rehabil Assist Technol. 2010;5(6):456-461.

17. Velik R, Hoffmann U, Zabaleta H, Marti Masso JF, Keller T. The effect of visual cues on the number and duration of freezing episodes in Parkinson's patients. Conf Proc IEEE Eng Med Biol Soc. 2012;2012: 4656-4659.

18. Jovanov E, Wang E, Verhagen L, Fredrickson M, Fratangelo R. Defoga real time system for detection and unfreezing of gait of Parkinson's patients. Conf Proc IEEE Eng Med Biol Soc. 2009;2009:5151-5154.

19. Moore ST, MacDougall HG, Ondo WG. Ambulatory monitoring of freezing of gait in Parkinson's disease. J Neurosci Methods. 2008; 167(2):340-348

20. Morris TR, Cho C, Dilda V, et al. A comparison of clinical and objective measures of freezing of gait in Parkinson's disease. Parkinsonism Relat Disord. 2012;18(5):572-577.

21. Liang D, Ivanov $\mathrm{K}, \mathrm{Li} \mathrm{H}$, et al. Exploration and comparison of the preimpact lead time of active and passive falls based on inertial sensors. Biomed Mater Eng. 2014;24(1):279-288.

22. Nieuwboer A, De Weerdt W, Dom R, Lesaffre E. A frequency and correlation analysis of motor deficits in Parkinson patients. Disabil Rehabil. 1998;20(4):142-150.

23. Snijders AH, Haaxma CA, Hagen YJ, Munneke M, Bloem BR. Freezer or non-freezer: clinical assessment of freezing of gait. Parkinsonism Relat Disord. 2012;18(2):149-154.

24. Lynn P, Fuerst W. The fast Fourier transforms - Basis of the FFT. In Introductory Digital Signal Processing. 2nd ed. John Wiley \& Sons; 1999:221-232.

25. Lynn P, Fuerst W. FFT Processing - Introduction. Introductory Digital Signal Processing. 2nd ed: John Wiley \& Sons; 1999:251-253.
Clinical Interventions in Aging

\section{Publish your work in this journal}

Clinical Interventions in Aging is an international, peer-reviewed journal focusing on evidence-based reports on the value or lack thereof of treatments intended to prevent or delay the onset of maladaptive correlates of aging in human beings. This journal is indexed on PubMed Central, MedLine,

\section{Dovepress}

CAS, Scopus and the Elsevier Bibliographic databases. The manuscript management system is completely online and includes a very quick and fair peer-review system, which is all easy to use. Visit http://www.dovepress. com/testimonials.php to read real quotes from published authors. 